\title{
LDPC Code Design for Fading Interference Channels
}

\author{
Mahdi Shakiba-Herfeh, Student Member, IEEE, A. Korhan Tanc ${ }^{(0)}$, Member, IEEE, \\ and Tolga M. Duman (1), Fellow, IEEE
}

\begin{abstract}
We focus on the two-user Gaussian interference channel (IC) with fading and study implementation of different encoding/decoding schemes with low-density parity-check (LDPC) codes for both quasi-static and fast fading scenarios. We adopt Han-Kobayashi encoding, derive stability conditions on the degree distributions of LDPC code ensembles, and obtain explicit and practical code designs. In order to estimate the decoding thresholds, a modified form of the extrinsic information transfer chart analysis based on binary erasure channel approximation for the incoming messages from the component LDPC decoders to state nodes is developed. The proposed code design is employed in several examples for both fast and quasi-static fading cases. Comprehensive set of examples demonstrate that the designed codes perform close to the achievable information theoretic limits. Furthermore, multiple antenna transmissions employing the Alamouti scheme for fading ICs are studied, a special receiver structure is developed, and specific codes are explored. Finally, advantages of the designed codes over point-to-point optimal ones are demonstrated via both asymptotic and finite block length simulations.
\end{abstract}

Index Terms-Low-density parity-check codes, interference channels, code design, iterative joint decoding, Han-Kobayashi encoding.

\section{INTRODUCTION}

I NTERFERENCE channel (IC) is used to model multiple one-to-one communicating pairs. In this model, each receiver observes its intended transmitter's signal, which is corrupted by unintended signals of the other transmitters, and it is assumed that there is no cooperation among any of the transmitters or receivers. Over the last few decades, a plethora of studies have been performed for an information theoretic characterization of the two-user IC, however, except for some special cases, the exact capacity region is still unknown. For the general case, the best inner bound is attained with the Han-Kobayashi (HK) [1] coding scheme. In this scheme, transmitters split their messages into public and private parts. The public messages are decoded by both receivers, while the private messages are decoded by the corresponding receivers only. On the other hand, a simple inner bound on the achievable rates over ICs is obtained by treating interference as noise (TIN) in which the receivers attempt to only decode their intended messages. At the opposite

Manuscript received July 21, 2018; revised November 12, 2018; accepted December 18, 2018. Date of publication January 9, 2019; date of current version March 14, 2019. This work was supported by The Scientific and Technological Research Council of Turkey (TUBITAK) under Grant 114E601. This paper was presented in part at the IEEE International Conference on Communications, Kansas City, Missouri, May 2018. The review of this paper was coordinated by Prof. Y. Li. (Corresponding author: A. Korhan Tanc.)

M. Shakiba-Herfeh and T. M. Duman are with the Department of Electrical and Electronics Engineering, Bilkent University Ankara 06800, Turkey (e-mail: herfeh@ee.bilkent.edu.tr; duman@ee.bilkent.edu.tr).

A. K. Tanc is with the Department of Biomedical Engineering, Biruni University Istanbul 34020, Turkey (e-mail: atanc@biruni.edu.tr).

Digital Object Identifier 10.1109/TVT.2019.2892036 extreme, another (simple) inner bound is obtained by simultaneously unique decoding (SUD), which refers to complete decoding of all the messages at both receivers. Each of these two approaches is optimal for a certain range of channel parameters [2], however, for the general case, they are not capacity achieving.

Despite a rich set of information theoretical results, there are only a limited number of investigations in the literature regarding practical (explicit and implementable) coding schemes for interference channels. In [3], the authors design low-density parity-check (LDPC) codes for the Gaussian IC (GIC) by assuming symmetric rate pairs and partial joint decoding. In [4], the authors present a concrete coding scheme to approach the theoretically achievable rate pairs by using LDPC codes concatenated with convolutional codes. Implementation of the HK coding scheme for a general GIC based on LDPC codes is studied in [5] where the authors perform decoding threshold analysis by utilizing Monte Carlo simulations, and show that with their proposed code design method, rate pairs close to the HK inner bounds can be attained. Furthermore, the authors in [6] propose a polar coding scheme for ICs, and show theoretically that fully joint decoder for the HK coding scheme can be simplified by employing partial joint decoders at the receivers, which is more favorable to polar coding. However, all of these studies are restricted to the case of fixed channel gains, and to the best of our knowledge, there is no work on code design for fading ICs in the literature.

Motivated by the relevance of fading ICs to wireless communications of multiple users utilizing the same spectrum, in this paper, we focus on designing practical coding schemes for the two-user IC with (fast and quasi-static) Rayleigh fading. In particular, due to their special features, we consider LDPC codes as the channel coding solution. LDPC codes are expected to be widely used in the 5th-generation wireless systems due to their adaptability to the new demands in wireless communications such as high throughput and wide range of block lengths (100-8 k information bits) and a variety of coding rates [7]. LDPC codes offer performance very close to the channel capacity for point-to-point (P2P) communications [8]. We also note that while there are many LDPC code constructions for fading channels [9], designs with joint decoding of interfering messages are very scarce. As LDPC code constructions are sensitive to the decoder architecture, new constructions are needed under joint decoding with a nonlinear state node. Towards this goal, the authors in [10] utilize LDPC codes in fast fading broadcast channels by employing superposition coding and joint decoding at the better receiver, and they show that the new designs perform close to the boundary of the achievable rate region. The need for new LDPC code designs for fading multiple-access channels (MACs) are also reported [11].

In our development of LDPC code design for fading ICs, we first elaborate on the information theoretical limits, and 
derive stability conditions for the adopted iterative decoder. We then utilize random perturbations for code search and perform decoding threshold analysis with extrinsic information transfer (EXIT) charts relying on a binary erasure channel (BEC) approximation for the state nodes. We perform code design for several instances of fast fading ICs. For SUD, we demonstrate a performance close to the asymptotic achievable signal-to-noise ratio (SNR) limits. With HK encoding, we design new code ensembles, which perform beyond the limits of TIN and SUD schemes clearly illustrating the need for HK based practical channel codes for fading ICs. For quasi-static fading ICs, we perform code designs for SUD and flexible decoding schemes, where the latter scheme refers to a system of receivers choosing the best strategy between TIN and SUD with respect to the observed channel gains. We demonstrate that our designed codes perform close to the outage limits of the corresponding coding schemes.

With the motivation that many wireless transceivers are equipped with multiple antennas, we also consider multiple antenna transmissions over quasi-static fading ICs. Specifically, we combine LDPC coding with the Alamouti scheme [12] to obtain spatial diversity for transmitters equipped with two antennas. We describe the structure of the receivers and perform LDPC code design for this scenario. We compare performance of the designed codes with P2P optimal off-the-shelf codes. The results demonstrate that there are significant improvements possible in the resulting frame error rates (FERs). Finally, we also focus on structured LDPC code constructions via protographs and design codes with low error floors.

The paper is organized as follows. In Section II, we describe the two-user fading interference channel model, and highlight the encoding and decoding alternatives. We also summarize the computation of outage rate regions for different schemes. In Section III, we derive stability conditions for the joint decoding of LDPC codes over fading ICs, and present a modified EXIT chart analysis to estimate the decoding thresholds of the code ensembles when two public and one private messages are jointly decoded. We also verify the accuracy of the method by using density evolution (DE). In Section IV, the encoding/decoding procedure is formulated for the case with two transmit antennas. In Section V, we detail the code optimization procedure, and in Section VI, we provide extensive simulation results with the newly designed codes over fading ICs. Finally, we conclude the paper in Section VII.

\section{SySTEM MODEL AND PRELIMINARIES}

A two-user fading IC is represented by the set of equations

$$
\begin{aligned}
& Y_{1}=h_{11} X_{1}+h_{21} X_{2}+z_{1}, \\
& Y_{2}=h_{12} X_{1}+h_{22} X_{2}+z_{2},
\end{aligned}
$$

where $X_{i}$ is the transmitted signal from transmitter $i$ subject to the power constraint $E\left\{\left|X_{i}\right|^{2}\right\} \leq P_{i}, h_{i j}$ is the complex channel gain between transmitter $i$ and receiver $j$, and $Y_{j}$ is the signal at receiver $j$, for $i, j=1,2$. The noise term $z_{j}$ is independent and identically distributed (i.i.d.) circularly symmetric complex Gaussian with zero mean and $\frac{N_{0}}{2}$ variance per dimension. We assume that the channel gains are (spatially independent) zero mean complex Gaussian random variables as well (i.e., Rayleigh fading). In the fast fading case, the channel coefficients change independently from one symbol to the next. On the other hand, in the quasi-static fading case, they remain constant during the

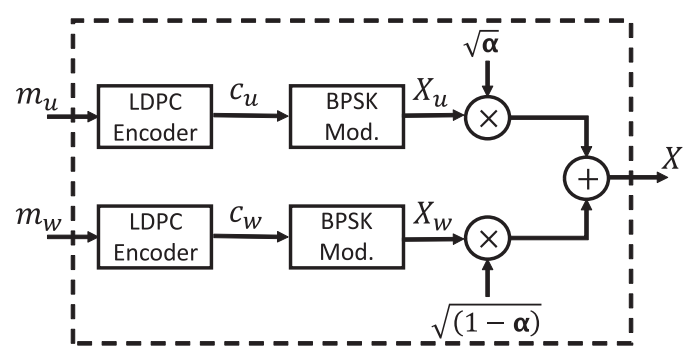

Fig. 1. The block diagram of the transmitter with HK encoding.

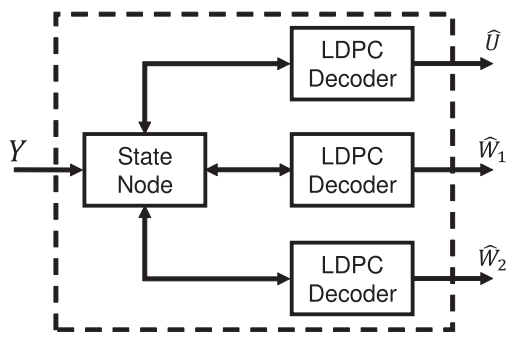

Fig. 2. The block diagram of the receiver with joint decoding.

entire codeword. We use the signal to noise ratio and interference to noise ratio definitions $S N R_{i}=E\left\{\frac{\left\|h_{i i}\right\|^{2} P_{i}}{N_{0}}\right\}$ and $I N R_{i}=$ $E\left\{\frac{\left\|h_{j i}\right\|^{2} P_{j}}{N_{0}}\right\}$ with $i, j=1,2, i \neq j$, respectively. Without loss of generality, we take $P_{1}=P_{2}=1$ and $N_{0}=1$.

With HK type encoding, the message is split into private and public parts at each transmitter. The private $\left(U_{i}\right)$ and public $\left(W_{i}\right)$ messages are separately encoded by the component LDPC codes and transmitted via binary phase shift keying (BPSK), i.e., 0 is transmitted as +1 and 1 is transmitted as -1 . The transmitter sends a linear superposition (sum) of the modulated signals. In other words,

$$
X_{i}=\sqrt{\alpha_{i}} X_{u_{i}}+\sqrt{1-\alpha_{i}} X_{w_{i}}, \quad i=1,2,
$$

where $\alpha \in[0,1]$ is a parameter employed for adjusting the power allocated to the private message. Fig. 1 shows a detailed block diagram of the transmitter under consideration.

At each receiver, we employ joint decoding with three component LDPC decoders and a state node as depicted in Fig. 2. In this setup, the log-likelihood ratios (LLRs) of the component decoders are exchanged via a state node in an iterative fashion. Initially, the a priori LLRs for the messages are calculated using the channel output and fed to the corresponding component decoders. At later iterations, the state node updates the a priori LLRs by utilizing both the channel output and the a posteriori LLRs from the component decoders, improving the iterative decoder's performance.

At receiver $j$, the outgoing LLR from the state node corresponding to the $i$ th bit of the message $k$ is calculated as [5]

$$
L\left(c_{k}(i), Y_{j}(i)\right)=\log \left(\frac{\sum_{C_{i} \in S_{i}^{k+}} f_{Y_{j}}\left(Y_{j}(i) \mid C_{i}\right) P\left(C_{i}\right)}{\sum_{C_{i} \in S_{i}^{k-}} f_{Y_{j}}\left(Y_{j}(i) \mid C_{i}\right) P\left(C_{i}\right)}\right),
$$

where $c_{k}(i)$ represents the $i$ th coded bit of the message, which can be public or the intended private one. $C_{i}$ is the vector consisting of the $i$ th bits of all the codewords, i.e., $C_{i}=\left\{c_{u_{1}}(i), c_{w_{1}}(i), c_{u_{2}}(i), c_{w_{2}}(i)\right\} . P\left(C_{i}\right)$ is the probability that $C_{i}$ is transmitted, and it is determined by the outputs of 
the component LDPC decoders. $f_{Y_{j}}$ is the probability density function (PDF) of $Y_{j}$, and $S_{i}^{k+}\left(S_{i}^{k-}\right)$ is the set of all codewords with $c_{k}(i)=0\left(c_{k}(i)=1\right)$.

Following [8], the variable and check node degree distributions of an ensemble of irregular LDPC codes are denoted by $\lambda(x)=\sum_{i=2}^{d_{v}} \lambda_{i} x^{i-1}$ and $\rho(x)=\sum_{i=2}^{d_{c}} \rho_{i} x^{i-1}$, respectively, where $d_{v}$ is the maximum variable node degree, and $d_{c}$ is the maximum check node degree. We assume that each receiver has the knowledge of the random channel gains contributing to its own received signal. Furthermore, power allocation parameters $\left(\alpha_{i}\right.$ 's) are known at both receivers.

\section{A. Outage Rate Region}

In this subsection, we explicitly identify the boundaries of the outage rate region for different decoding schemes, which refers to the set of rate vectors such that the outage constraints are satisfied [13]. We call an event as outage when at least one of the users fails to decode the packets with its decoding policy. We want an outage event probability of less than $P_{o}$. Noting that the outage rate region is the set of all rate pairs falling inside the achievable rate region (ARR) (which is a function of the channel gains, hence random) with probability higher than $1-P_{o}$, we elaborate on its computation for different decoding schemes in the following.

1) Without Rate Splitting: We first assume that the transmitters do not split their messages, i.e., they only use one codeword. The messages can be considered as either private or public as explained below.

TIN decoding scheme: In this scheme, each receiver only decodes its own message and treats the interference signal as noise. Hence, the receivers can decode their own messages if

$$
\begin{aligned}
& R_{1}<I\left(X_{1} ; Y_{1}\right), \\
& R_{2}<I\left(X_{2} ; Y_{2}\right),
\end{aligned}
$$

where $I(X ; Y)$ stands for the mutual information of $X$ and $Y$.

SUD scheme: In this scheme, the receivers decode both messages, i.e., this set-up can be considered as a compound MAC. Therefore, the messages can be successfully decoded at the receivers if

$$
\begin{aligned}
& R_{1}<\min \left\{I\left(X_{1} ; Y_{1} \mid X_{2}\right), I\left(X_{1} ; Y_{2} \mid X_{2}\right)\right\} \\
& R_{2}<\min \left\{I\left(X_{2} ; Y_{1} \mid X_{1}\right), I\left(X_{2} ; Y_{2} \mid X_{1}\right)\right\} \\
& R_{1}+R_{2}<\min \left\{I\left(X_{1}, X_{2} ; Y_{1}\right), I\left(X_{1}, X_{2} ; Y_{2}\right)\right\} .
\end{aligned}
$$

Flexible decoding scheme: Since the random channel gains are known by the receivers in our model, they can choose their own decoding strategy between TIN and SUD with respect to the observed channel realizations. Hence, the receivers can decode their own messages if

$$
\left(R_{1}, R_{2}\right) \in\left(\mathcal{R}_{T I N 1} \cup \mathcal{R}_{S U D 1}\right) \cap\left(\mathcal{R}_{T I N 2} \cup \mathcal{R}_{S U D 2}\right),
$$

where $\mathcal{R}_{T I N j}$ (and $\mathcal{R}_{S U D j}$ ) represents the TIN-ARR (and SUD-ARR) at receiver $j$. Fig. 3 demonstrates the decoding regions of the above schemes for a specific set of channel parameters.

2) With Rate Splitting: In this case, we assume that the transmitters split their messages with a fixed rate into two messages. The channel can be modeled as a compound $K$-user MAC, with respect to the decoding scheme, and the ARR for each scheme can be obtained as follows.

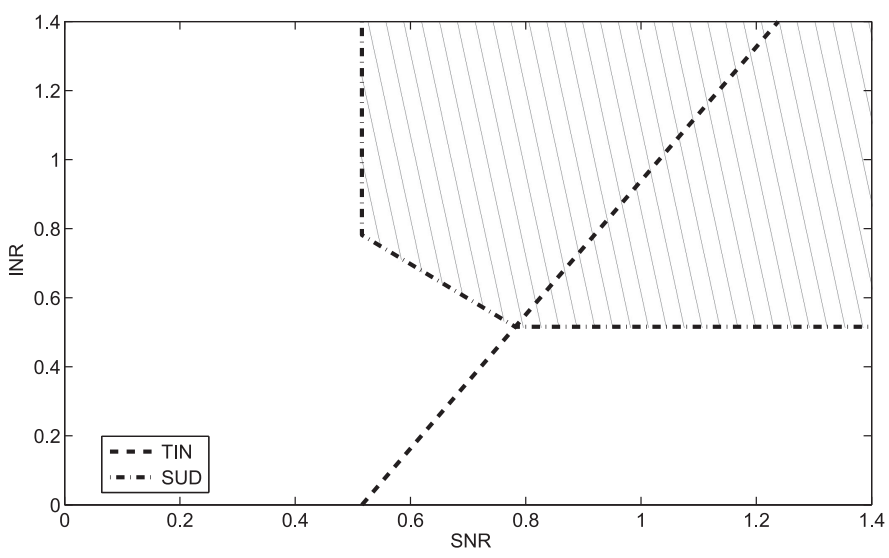

Fig. 3. An example of decodable region for TIN and SUD schemes without rate splitting at receiver 1 for an unfaded channel with Gaussian inputs and rate pair $\left(R_{1}, R_{2}\right)=(0.6,0.6)$. The decodable region of the flexible decoding is the union of the decodable regions of TIN and SUD schemes.

TIN decoding scheme: In this scheme each receiver decodes both messages of the intended transmitter and treat the interfering signals from the unintended transmitter as noise. Hence, the ARR is characterized by ${ }^{1}$ (for $i, j=0,1$ )

$$
\begin{aligned}
& i R_{u_{1}}+j R_{w_{1}}<I\left(i X_{u_{1}}, j X_{w_{1}} ; Y_{1} \mid(1-i) X_{u_{1}},(1-j) X_{w_{1}}\right), \\
& i R_{u_{2}}+j R_{w_{2}}<I\left(i X_{u_{2}}, j X_{w_{2}} ; Y_{2} \mid(1-i) X_{u_{2}},(1-j) X_{w_{2}}\right) .
\end{aligned}
$$

SUD scheme: In this scheme each receiver decodes all four messages of the transmitters. The receivers are able to decode successfully if (7), shown at the bottom of the next page, is satisfied.

HK decoding scheme: The receivers decode the two messages of the intended transmitter and only the second message of the unintended transmitter. Hence, they are successful if (8), shown at the bottom of the next page, is satisfied. Flexible decoding scheme: Similar to the previous case of no rate splitting, the receivers can choose their decoding policy with respect to the instantaneous channel gains. Hence, they are able to decode their own messages by utilizing one of the TIN, SUD and HK schemes. Hence, we have

$$
\begin{aligned}
\left(R_{1}, R_{2}\right) \in\left(\mathcal{R}_{T I N 1} \cup \mathcal{R}_{S U D 1} \cup \mathcal{R}_{H K 1}\right) \\
\cap\left(\mathcal{R}_{T I N 2} \cup \mathcal{R}_{S U D 2} \cup \mathcal{R}_{H K 2}\right),
\end{aligned}
$$

where $\mathcal{R}_{T I N j}, \mathcal{R}_{S U D j}$, and $\mathcal{R}_{H K j}$ represent the TIN-ARR, SUD-ARR, and HK-ARR at receiver $j$, respectively. Fig. 4 indicates the rate regions with the specified decoding approaches for a specific set of channel parameters. As observed in Figs. 3 and 4, the decodable region of flexible decoding scheme changes by splitting the messages. We deduce that splitting messages may be favorable or not depending on the fading realizations.

\section{PERFORMANCE ANALYSIS}

In this section, our objective is to find the decoding thresholds of the candidate LDPC code ensembles in the code optimization

\footnotetext{
${ }^{1}$ We use this notation for compactness. Note that $i$ and $j$ cannot be equal to zero at the same time.
} 


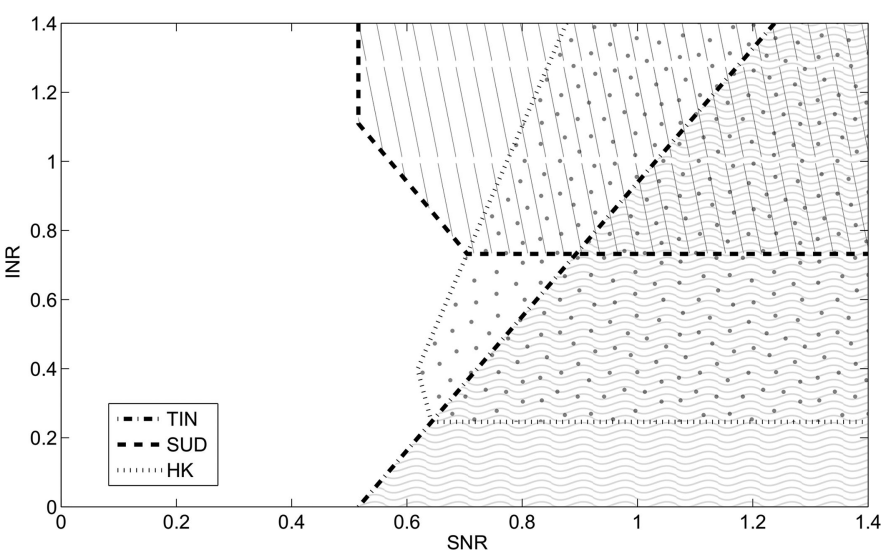

Fig. 4. An example of decodable region at receiver one for TIN, SUD, and HK decoding scheme and unfaded channel with Gaussian inputs and rate splitting with $\left(R_{u_{1}}, R_{w_{1}}\right)=(0.3,0.3),\left(R_{u_{2}}, R_{w_{2}}\right)=(0.45,0.15)$, and $\left(\alpha_{1}, \alpha_{2}\right)=$ $(0.5,0.5)$. The decodable region of the flexible decoding is the union of the decodable regions of TIN, SUD, and HK decoding.

process. For this purpose, we first derive the stability conditions for both fast and quasi-static fading scenarios, which give lower bounds on the decoding threshold. We then introduce a modified version of the EXIT analysis to estimate the decoding thresholds upon which the code design procedure is based.

\section{A. Stability Conditions}

Stability condition characterizes the convergence behavior of iterative decoders under the assumption that a low error probability has already been achieved. It is essentially utilized for eliminating error floors [14], and it has been studied previously for joint iterative decoding in multiuser scenarios [10] and [5] for broadcast and unfaded IC with real channel gains, respectively. Here, we extend the results to fading ICs under joint iterative decoding. We assume asymptotic conditions, which impose cycle free LDPC codes and almost zero decoding error, and via small perturbations of the degree distribution, we investigate the behavior of the joint decoder.

1) All Public Scheme: In this special case of the HK encoding scheme, both messages are decoded at both receivers, i.e., this case can be considered as a compound MAC. As it is shown in [14], in MAC channels, in order to find the stability condition on the degree distributions of the LDPC codes used, one can assume that the other message has been decoded completely and its effect is subtracted from the channel observations. Hence, the condition boils down to two single user stability conditions, and the modified channel becomes $Y_{j}^{\prime}=h_{i j} X_{w_{i}}+z_{j}$. By applying the stability condition of the P2P channel to the compound MAC, the stability condition for the degree distributions of public messages for a fast fading IC is expressed as follows:

$$
\frac{1}{\lambda_{w_{i}}^{\prime}(0) \rho_{w_{i}}^{\prime}(1)}>\max \left\{E_{h_{i i}}\left[e^{\left(-\left\|h_{i i}\right\|^{2}\right)}\right], E_{h_{i j}}\left[e^{\left(-\left\|h_{i j}\right\|^{2}\right)}\right]\right\},
$$

for $i, j=1,2$ and $i \neq j$. Here $\lambda_{i}^{\prime}(0)$ denotes the first derivative of the variable node degree distribution polynomial $\lambda(x)$ at $x=0$, and $\rho_{i}^{\prime}(1)$ denotes the first derivative of the check node degree distribution polynomial $\rho(x)$ at $x=1$, respectively. $E_{h_{i i}}$ $\left(E_{h_{i j}}\right)$ indicates expectation with respect to the random variable $h_{i i}\left(h_{i j}\right)$.

Following a similar approach, we derive the stability condition for the quasi-static channel as well. ${ }^{2}$ In this case since we work with an outage probability formulation, the codewords should be decoded error free with a probability higher than $1-P_{o}$. Also, as it is discussed above, the modified channel for the SUD case is similar to a P2P channel. We first define a level $H$ such that, with probability $1-P_{o}$ the channel power is higher than this threshold. Then, we introduce the stability condition as:

$$
\frac{1}{\lambda_{w_{i}}^{\prime}(0) \rho_{w_{i}}^{\prime}(1)}>\max \left\{e^{\left(-H_{i i}{ }^{2}\right)}, e^{\left(-H_{i j}{ }^{2}\right)}\right\}
$$

for $i, j=1,2$ and $i \neq j$. Here $H_{i i}$ and $H_{i j}$ should satisfy the condition that the union of four events $\bigcup_{i, j}\left\{H_{i j} \geq\left\|h_{i j}\right\|\right\}$ has probability less than or equal to $P_{o}$.

2) The HK Scheme: In this case, each receiver decodes both public messages and its intended private message, however, the unintended private message is not decoded. Therefore, to derive the stability condition, we assume that the other two messages of interest have been completely decoded and the unintended message is treated as noise. Using (1) and the superposition formulation, the modified channel outputs $Y_{j}^{\prime}$ and $Y_{j}^{\prime \prime}$ for the public and intended private messages are obtained by cancelling out the completely decoded messages:

$$
\begin{aligned}
Y_{j}^{\prime} & =\sqrt{1-\alpha_{k}} h_{k j} X_{w_{k}}+\sqrt{\alpha_{i}} h_{i j} X_{u_{i}}+z_{j}, \\
Y_{j}^{\prime \prime} & =\sqrt{\alpha_{j}} h_{j j} X_{u_{j}}+\sqrt{\alpha_{i}} h_{i j} X_{u_{i}}+z_{j},
\end{aligned}
$$

for $i, j, k=1,2$ and $i \neq j$. Eq. (12) expresses the modified channel outputs for the public message of user $k$ at receiver $j$. In order to calculate the stability condition, we employ an

\footnotetext{
${ }^{2}$ However, different from the fast fading case, the stability condition for the quasi-static fading scenario serves as a looser lower bound on the decoding threshold. Hence, we only utilize it just for speeding up our design procedure. For eliminating error floors, we employ a robust design algorithm as explained in Section V.
}

$$
\begin{aligned}
& i R_{u_{1}}+j R_{w_{1}}+k R_{u_{2}}+l R_{w_{2}}<I\left(i X_{u_{1}}, j X_{w_{1}}, k X_{u_{2}}, l X_{w_{2}} ; Y_{1} \mid(1-i) X_{u_{1}},(1-j) X_{w_{1}},(1-k) X_{u_{2}},(1-l) X_{w_{2}}\right), \\
& i R_{u_{1}}+j R_{w_{1}}+k R_{u_{2}}+l R_{w_{2}}<I\left(i X_{u_{1}}, j X_{w_{1}}, k X_{u_{2}}, l X_{w_{2}} ; Y_{2} \mid(1-i) X_{u_{1}},(1-j) X_{w_{1}},(1-k) X_{u_{2}},(1-l) X_{w_{2}}\right) \\
& i, j, k, l=0,1 \\
& i R_{u_{1}}+j R_{w_{1}}+k R_{w_{2}}<I\left(i X_{u_{1}}, j X_{w_{1}}, k X_{w_{2}} ; Y_{1} \mid(1-i) X_{u_{1}},(1-j) X_{w_{1}},(1-k) X_{w_{2}}\right) \\
& i R_{u_{2}}+j R_{w_{1}}+k R_{w_{2}}<I\left(i X_{u_{2}}, j X_{w_{1}}, k X_{w_{2}} ; Y_{2} \mid(1-i) X_{u_{2}},(1-j) X_{w_{1}},(1-k) X_{w_{2}}\right) \\
& i, j, k=0,1
\end{aligned}
$$


auxiliary function $f$ given by (14), shown at the bottom of the page, where $E_{N_{1}, N_{2}}$ indicates expectation with respect to the random variables $N_{1}, N_{2} \sim \mathcal{N}\left(0, \frac{1}{2}\right)$, as derived in the Appendix. In fact, (14) is the Bhattacharyya constant of the channel $f_{Y \mid s}(y) \sim h_{1} s+h_{2} X+N$, where $X \in\{ \pm 1\}$ equally likely and the channel gains are complex and constant. Thus, we obtain the following inequality for the intended private message over a fast fading IC

$$
\frac{1}{\lambda_{u_{i}}^{\prime}(0) \rho_{u_{i}}^{\prime}(1)}>E_{h_{i i}, h_{j i}}\left[f\left(\sqrt{\alpha_{i}} h_{i i}, \sqrt{\alpha_{j}} h_{j i}\right)\right]
$$

for $i, j=1,2$ and $i \neq j$. Similarly, the stability condition for the public messages is

$$
\begin{array}{r}
\frac{1}{\lambda_{w_{i}}^{\prime}(0) \rho_{w_{i}}^{\prime}(1)}>\max \left(E_{h_{i i}, h_{j i}}\left[f\left(\sqrt{1-\alpha_{i}} h_{i i}, \sqrt{\alpha_{j}} h_{j i}\right)\right],\right. \\
\left.E_{h_{i j}}\left[f\left(\sqrt{1-\alpha_{i}} h_{i j}, \sqrt{\alpha_{i}} h_{i j}\right)\right]\right),
\end{array}
$$

for $i, j=1,2$ and $i \neq j$. The stability conditions for quasi-static fading interference channels for the private messages can be obtained as

$$
\frac{1}{\lambda_{u_{i}}^{\prime}(0) \rho_{u_{i}}^{\prime}(1)}>S_{u_{i}}
$$

where $S_{u_{i}}$ should satisfy $\operatorname{Pr}\left\{\bigcup_{i}\left\{S_{u_{i}} \leq f\left(\sqrt{\alpha_{i}} h_{i i}, \sqrt{\alpha_{j}} h_{j i}\right)\right\}\right\}$ $\leq P_{o}$ for $i, j=1,2$ and $i \neq j$. On the other hand, the stability condition for public messages can be expressed as

$$
\frac{1}{\lambda_{w_{i}}^{\prime}(0) \rho_{w_{i}}^{\prime}(1)}>\max \left(S_{1, w_{i}}, S_{2, w_{i}}\right) \text {. }
$$

Here $S_{1, w_{i}}$ and $S_{2, w_{i}}$ should satisfy the condition that $\operatorname{Pr}\left\{\bigcup_{i} E_{i}\right\} \leq P_{o}$ where the event $E_{i}$ is defined as $\left\{S_{1, w_{i}} \leq\right.$ $\left.f\left(\sqrt{1-\alpha_{i}} h_{i i}, \sqrt{\alpha_{j}} h_{j i}\right)\right\} \bigcup\left\{S_{2, w_{i}} \leq f\left(\sqrt{1-\alpha_{i}} h_{i j}, \sqrt{\alpha_{i}} h_{i j}\right)\right\}$ for $i, j=1,2$ and $i \neq j$.

\section{B. Modified EXIT Chart Analysis}

EXIT chart analysis is a prominent technique for estimating the decoding thresholds of LDPC code ensembles [15]. In this technique, the exchanged LLRs are assumed to have symmetric Gaussian distributions with a single parameter. Hence, the mutual information between the transmitted bits and the LLRs passed to the check nodes can be calculated as

$$
I_{v \rightarrow c}=\sum_{i} \lambda_{i} J\left((i-1) J^{-1}\left(I_{c \rightarrow v}\right)+J^{-1}\left(I_{s \rightarrow v}\right)\right),
$$

where $I_{c \rightarrow v}$ and $I_{s \rightarrow v}$ represent the mutual informations of the LLRs from the check nodes to the variable nodes and the mutual informations of the LLRs from the state nodes to the variable nodes, respectively. $J(x)$ is defined as

$$
J(x)=1-\int_{-\infty}^{\infty} \frac{1}{\sqrt{2 \pi x}} \exp \left(-\frac{\left(l-\frac{x}{2}\right)^{2}}{2 x}\right) \log _{2}\left(1+e^{-l}\right) d l .
$$

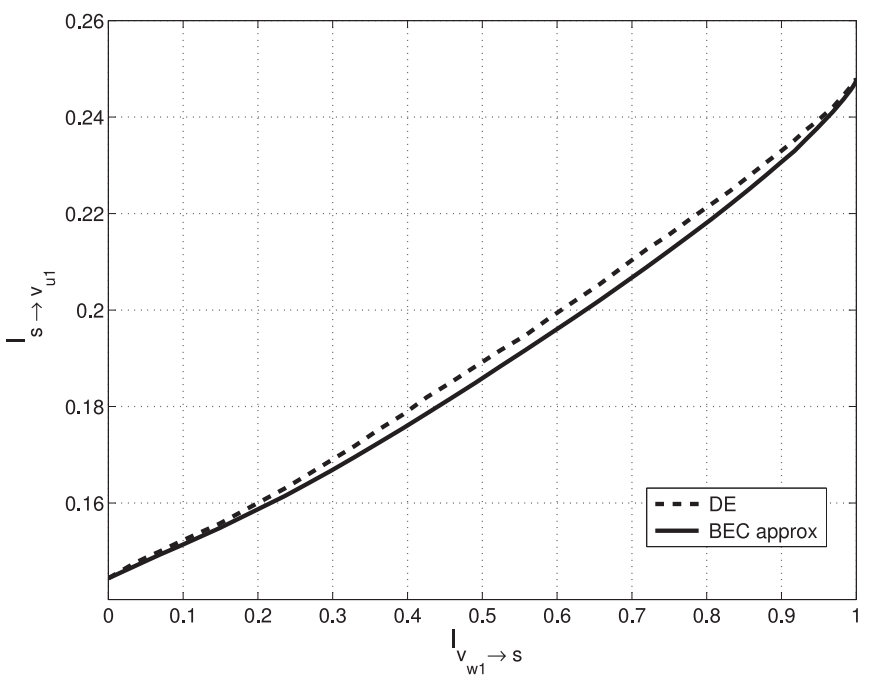

Fig. 5. Evolution of private message at the state node for the LDPC code ensembles given in Table $\mathrm{V}$ in $[5]$ with rate $(0.307,0.258)$ and channel with $I\left(X_{u} ; Y\right)=0.1444$ and $I\left(X_{u}, Y \mid X_{w_{1}}, X_{w_{2}}\right)=0.2477$.

In addition, the mutual information of the outgoing LLRs from the check nodes to the variable nodes can be calculated as

$$
\begin{aligned}
& I_{c \rightarrow v}=\sum_{i} \rho_{i}\left(1-J\left((i-1) J^{-1}\left(1-I_{v \rightarrow c}\right)\right)\right), \\
& I_{v \rightarrow s}=\sum_{i} \tilde{\lambda}_{i} J\left(i J^{-1}\left(I_{c \rightarrow v}\right)\right),
\end{aligned}
$$

where $\tilde{\lambda}(x)$ stands for the variable node degree distribution polynomial from the node perspective [11]. We model the links for incoming LLRs from component LDPC decoders as BECs with erasure probability $1-I_{v \rightarrow s}$, as employed in [10] for joint decoding of two messages. Via simulations it is observed the BEC approximation is suitable for estimating the decoding thresholds when multiple messages are jointly decoded (see Fig. 5). Under this BEC modeling, with probability $I_{v_{a} \rightarrow s}$ the value of the interfering signal $a$ is known by the state node, and with probability $1-I_{v_{a} \rightarrow s}$ there is no information about its value. By averaging over all the possibilities on the knowledge of the interfering signals, $I_{s \rightarrow v}$ for the state node connected to three component LDPC decoders can be obtained as

$$
\begin{aligned}
I_{s \rightarrow v}= & I_{v_{a} \rightarrow s} I_{v_{b} \rightarrow s} I\left(X ; Y \mid X_{a}, X_{b}\right) \\
& +\left(1-I_{v_{a} \rightarrow s}\right) I_{v_{b} \rightarrow s} I\left(X ; Y \mid X_{b}\right) \\
& +\left(1-I_{v_{b} \rightarrow s}\right) I_{v_{a} \rightarrow s} I\left(X ; Y \mid X_{a}\right) \\
& +\left(1-I_{v_{a} \rightarrow s}\right)\left(1-I_{v_{b} \rightarrow s}\right) I(X ; Y),
\end{aligned}
$$

where $a$ and $b$ indicate the other two component LDPC decoders connected to the state node.

We note that the above analysis can be generalized to the case of $K$ users with $K>2$ as well. To generalize the stability

$f\left(h_{1}, h_{2}\right)=E_{N_{1}, N_{2}}\left[\left(e^{\left(-\left\|h_{1}\right\|^{2}-\left\|h_{2}\right\|^{2}\right)}\right) \times \sqrt{\frac{\cosh \left(4 N_{1} \operatorname{Re}\left(h_{2}\right)+4 N_{2} \operatorname{Im}\left(h_{2}\right)\right)+\cosh \left(4 \operatorname{Re}\left(h_{1}\right) \operatorname{Re}\left(h_{2}\right)+4 \operatorname{Im}\left(h_{1}\right) \operatorname{Im}\left(h_{2}\right)\right)}{2}}\right]$. 


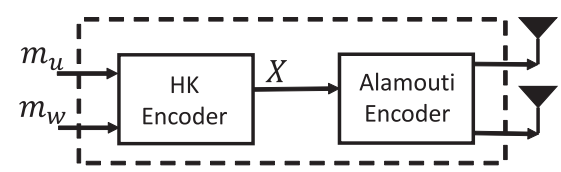

Fig. 6. The block diagram of the transmitter for Alamouti technique.

condition analysis, the required maximizations for public messages given in (10), (11), (16) and (18) should be extended $K$ terms. In addition, the introduced $f$ function in (14) can be modified as a $K$ dimensional function. Regarding the modified EXIT chart analysis, the BEC approximation for the output of the state node should contain $2^{K}$ additive terms.

\section{Multiple Antenna Transmission}

One of the techniques to combat undesirable effects of fading is to construct different links between the transmitter and the receiver by employing multiple antennas, which can be used to obtain spatial diversity. With this motivation, in this section, we consider fading ICs when the transmitters are equipped with multiple antennas. Specifically, we address LDPC code optimization for quasi-static fading ICs with two transmit antennas and one receive antenna for each user. In the following, we design the corresponding transmitter and receiver structures, and provide a formulation of the messages passed between different decoding blocks.

The block diagram of the transmitter with two antennas is depicted in Fig. 6. The output symbols of the HK encoder are fed to an Alamouti encoder. Two symbols are considered at a time, say $x_{a}$ and $x_{b}$, and they are transmitted in two consecutive time slots. In the first time slot, the first antenna transmits the symbol $x_{a}$ and the second antenna transmits the symbol $x_{b}$. In the second time slot, the first antenna transmits $-x_{b}^{*}$ and the second antenna transmits $x_{a}^{*}$.

We now formulate the SUD scheme corresponding to the above Alamouti based transmission. We set $E\left\{\left|X_{i}\right|^{2}\right\} \leq \frac{P_{i}}{2}$ to satisfy the total power constraint. The channel observation for receiver 1 can be expressed as (23) where $h_{i}^{k}[n]$ represents the channel gain between the $k$ th antenna of user $i$ and receiver 1 at time $n$.

$$
\begin{aligned}
Y[2 n]= & h_{1}^{1}[2 n] X_{1}[2 n]+h_{1}^{2}[2 n] X_{1}[2 n+1] \\
& +h_{2}^{1}[2 n] X_{2}[2 n]+h_{2}^{2}[2 n] X_{2}[2 n+1]+z[2 n], \\
Y[2 n+1]= & h_{1}^{2}[2 n] X_{1}^{*}[2 n]-h_{1}^{1}[2 n] X_{1}^{*}[2 n+1] \\
& +h_{2}^{2}[2 n] X_{2}^{*}[2 n]-h_{2}^{1}[2 n] X_{2}^{*}[2 n+1]+z[2 n+1] .
\end{aligned}
$$

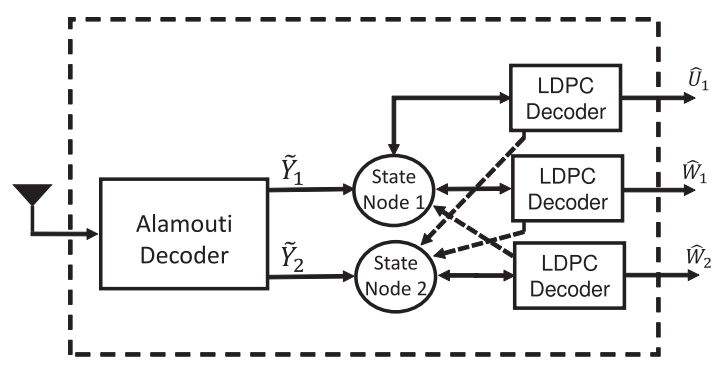

Fig. 7. The block diagram of the receiver 1 for Alamouti transmission scheme.

The decoder produces the combined signals (see Fig. 7)

$$
\begin{aligned}
\tilde{Y}_{1}[2 n] & =h_{1}^{1^{*}}[2 n] Y[2 n]+h_{1}^{2}[2 n] Y^{*}[2 n+1], \\
\tilde{Y}_{1}[2 n+1] & =h_{1}^{2^{*}}[2 n] Y[2 n]-h_{1}^{1}[2 n] Y^{*}[2 n+1],
\end{aligned}
$$

and

$$
\begin{aligned}
\tilde{Y}_{2}[2 n] & =h_{2}^{1^{*}}[2 n] Y[2 n]+h_{2}^{2}[2 n] Y^{*}[2 n+1], \\
\tilde{Y}_{2}[2 n+1] & =h_{2}^{2^{*}}[2 n] Y[2 n]-h_{2}^{1}[2 n] Y^{*}[2 n+1] .
\end{aligned}
$$

Notice that (24) can be written as (26), shown at the bottom of this page. It is clear that only the signal corresponding to the $n$th bit of the message of user 1 appears in $\tilde{Y}_{1}[n]$. Applying the same procedure, we can obtain $\tilde{Y}_{2}$ similarly. As shown in Fig. 7, the decoder outputs $\tilde{Y}_{1}$ and $\tilde{Y}_{2}$ are fed to the state nodes 1 and 2, respectively. Then the state nodes calculate the LLRs for the component decoders using their incoming LLRs and the decoder outputs. The calculated LLRs are exchanged between the state nodes and the component LDPC decoders in an iterative fashion.

It is evident from these formulations that after passing the received signal through the decoder for the Alamouti based transmission, the distribution of the coefficients of the intended and unintended signals are not complex Gaussian anymore, hence, new code designs may be needed to improve the system performance. We also note that the analysis developed above can be extended to the case of HK based coding as well.

We now derive a stability condition for the SUD scheme with Alamouti based transmission. We assume that the other message has completely been decoded. Hence, with respect to (26), the modified channel for user $i$ at receiver $j$ becomes $Y_{j}^{\prime}=$ $\left(\left\|h_{i j}^{1}\right\|^{2}+\left\|h_{i j}^{2}\right\|^{2}\right) X_{w_{i}}+\sqrt{\left\|h_{i j}^{1}\right\|^{2}+\left\|h_{i j}^{2}\right\|^{2}} z_{j}$. Similar to the single antenna case, the stability conditions for the degree distributions of public messages over a quasi-static fading interference channel can then be obtained as

$$
\frac{1}{\lambda_{w_{i}}^{\prime}(0) \rho_{w_{i}}^{\prime}(1)}>\max \left\{e^{\left(-H_{i i^{2}}\right)}, e^{\left(-H_{i j}{ }^{2}\right)}\right\}
$$

$$
\begin{aligned}
& \tilde{Y}_{1}[2 n]=\left(\left\|h_{1}^{1}[2 n]\right\|^{2}+\left\|h_{1}^{2}[2 n]\right\|^{2}\right) X_{1}[2 n]+\left(h_{1}^{1^{*}}[2 n] h_{2}^{1}[2 n]+h_{1}^{2}[2 n] h_{2}^{2^{*}}[2 n]\right) X_{2}[2 n] \\
& +\left(h_{1}^{1^{*}}[2 n] h_{2}^{2}[2 n]-h_{1}^{2}[2 n] h_{2}^{1^{*}}[2 n]\right) X_{2}[2 n+1]+h_{1}^{1^{*}}[2 n] z[2 n]+h_{1}^{2}[2 n] z^{*}[2 n+1], \\
& \tilde{Y}_{1}[2 n+1]=\left(\left\|h_{1}^{1}[2 n]\right\|^{2}+\left\|h_{1}^{2}[2 n]\right\|^{2}\right) X_{1}[2 n+1]+\left(h_{1}^{2^{*}}[2 n] h_{2}^{1}[2 n]-h_{1}^{1}[2 n] h_{2}^{2^{*}}[2 n]\right) X_{2}[2 n] \\
& +\left(h_{1}^{2^{*}}[2 n] h_{2}^{2}[2 n]+h_{1}^{1}[2 n] h_{2}^{1^{*}}[2 n]\right) X_{2}[2 n+1]+h_{1}^{2^{*}}[2 n] z[2 n]-h_{1}^{1}[2 n] z^{*}[2 n+1] .
\end{aligned}
$$


for $i, j=1,2$ and $i \neq j$. Here $H_{i i}$ and $H_{i j}$ should satisfy the condition that the union of four events $\bigcup_{i, j}\left\{H_{i j} \geq \frac{\left\|h{ }_{i j}^{1}\right\|^{2}+\left\|h_{i j}^{2}\right\|^{2}}{2}\right\}$ has probability less than or equal to $P_{o}$. Comparing this result with the single-antenna case in (11), we observe that the new stability condition takes into account the arithmetic mean of the channel powers.

\section{LDPC CODE OPTIMIZATION}

The proposed LDPC code optimization procedure relies on both the modified EXIT chart analysis and the derived stability condition. While the former is utilized for analyzing the asymptotic decoding performance of the code ensembles, the latter is used for eliminating code ensembles that can potentially produce high error floors. For fast fading IC with specific $S N R$ and $I N R$ values, we initialize the code optimization with $\mathrm{P} 2 \mathrm{P}$ optimal code ensembles. We generate new code ensembles using random perturbations, and we aim at improving the code rates while satisfying the stability condition and providing successful decoding. Once we improve upon the code rates, we assign the corresponding code ensembles as the updated ones, and we continue the evolution. The whole procedure is terminated when the code rates cannot be further improved [16].

Different from the fast fading case, in the quasi-static fading scenario, we consider a large number of channel realizations (taken as $2 \times 10^{4}$ in the numerical results) for checking the code ensembles under consideration. For each channel realization, we analyze the ARR, stability condition and modified EXIT in order. If at least one of the analyses fails, we declare the decoding for that channel realization as failure. If the overall failure rate is below $P_{o}$, we assign the corresponding code ensembles as the updated ones. Here we note that our aim is to reach an almost zero bit error rate (BER) for the fast fading case. However, for the quasi-static fading case, we aim at an FER less than or equal to $P_{o}$.

In each perturbation, we generate code ensembles with a specific rate increment $\Delta R$. For this purpose, we perturb both the check node and the variable node polynomials. The $i$ th coefficient of the check node polynomial is perturbed as $\bar{\rho}_{i}=$ $\rho_{i}+e_{c i}$, where $e_{c i}$ is the $i$ th element of the perturbing vector $\mathbf{e}_{c}$. We assume $e_{c i}=0$ if $\rho_{i}=0$. We notice that the perturbing vector should satisfy $\sum_{i} \bar{\rho}_{i}=1$, which results in

$$
\sum_{i} e_{c i}=0
$$

In order to control the amount of perturbation we impose

$$
\sigma_{e}^{2}=\sum_{i} e_{c i}^{2}
$$

For the variable node polynomial, we employ a perturbing vector $\mathbf{e}_{v}$, which satisfies (28) and (29) as well. Let $\Delta r_{1}$ be the rate increment after perturbing the check node polynomial. In order to have an overall rate increment of $\Delta R$, the increment after perturbing the variable node polynomial should be $\Delta r_{2}=\Delta R-\Delta r_{1}$. This enforces an extra constraint on $\mathbf{e}_{v}$ as

$$
\sum_{i} \frac{e_{v_{i}}}{i}=\frac{\Delta r_{2} \sum_{i} \frac{\bar{\rho}_{i}}{i}}{\left(1-\left(R_{0}+\Delta r_{1}\right)\right)^{2}-\Delta r_{2}\left(1-\left(R_{0}+\Delta r_{1}\right)\right)}
$$

where $R_{0}$ is the initial rate before perturbation. It follows that, two elements of $\mathbf{e}_{c}$ and three elements of $\mathbf{e}_{v}$ are dependent

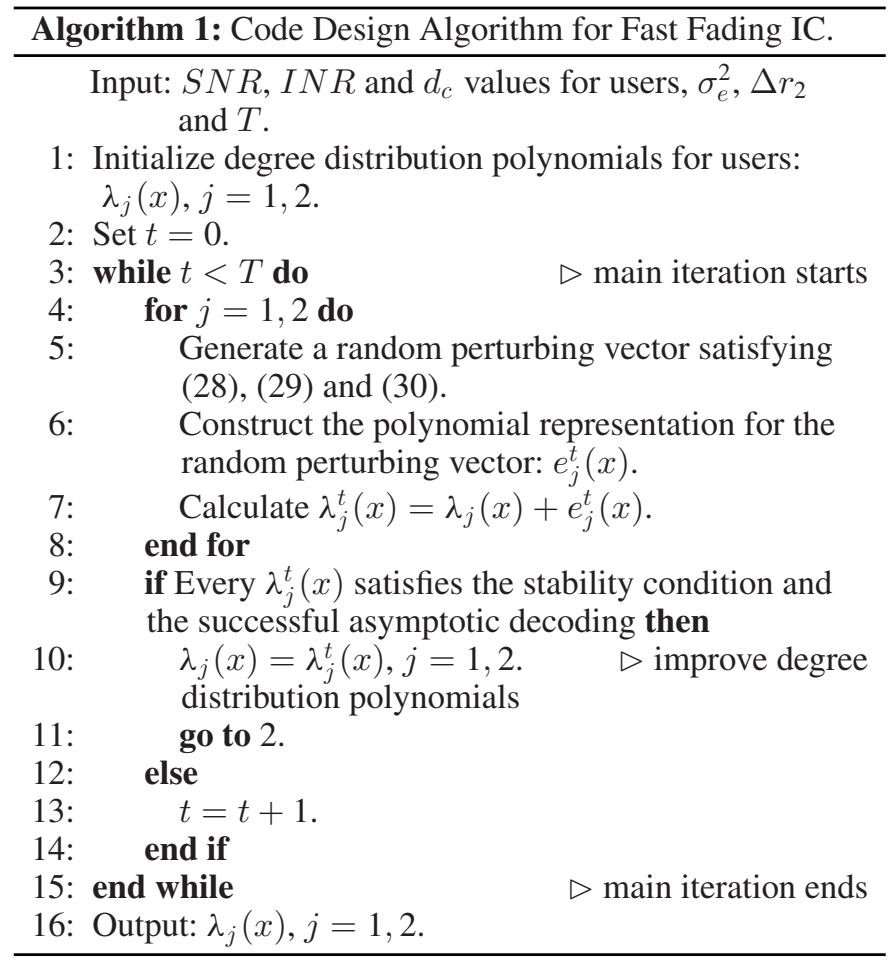

coefficients. The remaining free coefficients are generated according to a normal distribution. For simplicity we assume singleton distribution for the check nodes, i.e., $\rho(x)=x^{d_{c}-1}$, and we provide the design procedure (with $\Delta r_{1}=0$ ) in Algorithm 1.

\section{NUMERICAL RESULTS}

In this section, we design LDPC codes for fast and quasi-static fading ICs, and investigate their performance via simulations. Similar to [5], we select the nonzero variable node degrees as $\{2,3,4,9,10,19,20,49,50\}$. We assume $\sigma_{e}^{2}=0.005$, $\Delta r_{2}=0.005 R_{0}$ and $T=1000$ in the code design. Unless otherwise stated, we consider single transmit antenna users. For comparisons we also simulate P2P codes which are optimized using the conventional EXIT chart analysis [16].

\section{A. Scenario I - Fast Fading Scenario}

In the first example, we consider the SUD scheme over a Rayleigh fading IC with parameters $S N R_{1}=-3 \mathrm{~dB}, S N R_{2}=$ $S N R_{1}+0.5 \mathrm{~dB}, I N R_{1}=S N R_{1}+0.75 \mathrm{~dB}$ and $I N R_{2}=$ $S N R_{1}+1.25 \mathrm{~dB}$. For this set of parameters, we perform code optimization and provide the resulting degree distributions in Table I. In Table II we provide the decoding thresholds of both optimized and P2P code ensembles calculated via Monte Carlo simulations. We also provide information theoretical achievable SNR limits calculated by adapting the simple approach developed in [17]. Notice that our designed code ensembles perform about $0.3 \mathrm{~dB}$ away from them. Furthermore, all of the codes perform beyond the achievable limits with TIN. In addition, more than $0.3 \mathrm{~dB}$ design advantage can be attained with respect to the $\mathrm{P} 2 \mathrm{P}$ code ensembles. For highly asymmetric rate pairs, however, we observe that the design advantage is small.

We now consider a $\mathrm{HK}$ coding scheme over a Rayleigh fading IC with parameters $S N R_{1}=-2.5 \mathrm{~dB}, S N R_{2}=S N R_{1}-$ 
TABLE I

OPTIMIZED DEGREE DISTRIBUTIONS FOR SUD UNDER FAST FADING

\begin{tabular}{|c|c|c|c|c|c|c|c|c|c|c|c|}
\hline Msg. & $\mathrm{R}$ & $d_{c}$ & $\lambda_{2}$ & $\lambda_{3}$ & $\lambda_{4}$ & $\lambda_{9}$ & $\lambda_{10}$ & $\lambda_{19}$ & $\lambda_{20}$ & $\lambda_{49}$ & $\lambda_{50}$ \\
\hline \hline$W_{1}$ & 0.394 & 8 & 0.172489 & 0.234395 & 0.035092 & 0.225446 & 0.006194 & 0.004258 & 0.024904 & 0.061975 & 0.235248 \\
\hline$W_{2}$ & 0.3518 & 7 & 0.222048 & 0.143843 & 0.115085 & 0.209143 & 0.025527 & 0.001216 & 0.035363 & 0.128079 & 0.119696 \\
\hline \hline$W_{1}$ & 0.3879 & 7 & 0.233243 & 0.10733 & 0.215338 & 0.115348 & 0.041379 & 0.038024 & 0.107015 & 0.034526 & 0.107795 \\
\hline$W_{2}$ & 0.3879 & 7 & 0.245681 & 0.100373 & 0.196623 & 0.115048 & 0.044966 & 0.044935 & 0.107476 & 0.072281 & 0.072617 \\
\hline \hline$W_{1}$ & 0.3674 & 7 & 0.226061 & 0.199873 & 0.066646 & 0.118809 & 0.096977 & 0.011083 & 0.013204 & 0.021915 & 0.24543 \\
\hline$W_{2}$ & 0.4163 & 8 & 0.217262 & 0.108635 & 0.16151 & 0.112415 & 0.099672 & 0.010327 & 0.001427 & 0.189408 & 0.099345 \\
\hline \hline$W_{1}$ & 0.31 & 6 & 0.261265 & 0.094317 & 0.214035 & 0.061744 & 0.104821 & 0.031201 & 0.076642 & 0.071036 & 0.084937 \\
\hline$W_{2}$ & 0.425 & 8 & 0.199757 & 0.191086 & 0.075019 & 0.123773 & 0.143391 & 0.005096 & 0.04852 & 0.023703 & 0.189655 \\
\hline
\end{tabular}

TABLE II

DECODING THRESHOLDS IN TERMS OF $S N R_{1}$ FOR SUD UNDER FAST FADING

\begin{tabular}{|c|c|c|c|c|}
\hline $\begin{array}{c}\text { Rate pair } \\
\left(R_{1}, R_{2}\right)\end{array}$ & $\begin{array}{c}\mathrm{P} 2 \mathrm{P} \\
\text { code ensemble }\end{array}$ & $\begin{array}{c}\text { Optimized } \\
\text { code ensemble }\end{array}$ & $\begin{array}{c}\text { Achievable } \\
\text { limit (SUD) }\end{array}$ & $\begin{array}{c}\text { Achievable } \\
\text { limit (TIN) }\end{array}$ \\
\hline$(0.394,0.352)$ & $-2.70 \mathrm{~dB}$ & $-2.72 \mathrm{~dB}$ & $-3.10 \mathrm{~dB}$ & $-2.02 \mathrm{~dB}$ \\
\hline$(0.388,0.388)$ & $-2.66 \mathrm{~dB}$ & $-2.84 \mathrm{~dB}$ & $-3.10 \mathrm{~dB}$ & $-2.15 \mathrm{~dB}$ \\
\hline$(0.367,0.416)$ & $-2.38 \mathrm{~dB}$ & $-2.76 \mathrm{~dB}$ & $-3.05 \mathrm{~dB}$ & $-2.06 \mathrm{~dB}$ \\
\hline$(0.310,0.425)$ & $-2.73 \mathrm{~dB}$ & $-2.73 \mathrm{~dB}$ & $-3.03 \mathrm{~dB}$ & $-1.85 \mathrm{~dB}$ \\
\hline
\end{tabular}

$0.5 \mathrm{~dB}, I N R_{1}=S N R_{1}-1 \mathrm{~dB}$ and $I N R_{2}=S N R_{1}-1.5 \mathrm{~dB}$. We give the optimized degree distributions in Table III, and provide the decoding thresholds of both optimized and P2P code ensembles in Table IV along with the information theoretical achievable SNR limits for various schemes. We observe that our designed code ensembles perform about $0.4 \mathrm{~dB}$ away from the achievable SNR limits with HK coding, and they operate beyond the achievable limits with TIN and SUD. In addition, more than $0.3 \mathrm{~dB}$ design advantage can be attained with respect to the $\mathrm{P} 2 \mathrm{P}$ codes, which indicate the clear need for designing new codes for fading ICs.

\section{B. Scenario II - Quasi-Static Fading Scenario}

In this subsection, we provide code design examples for quasistatic fading ICs with different decoding approaches. We observe that, in general, rate splitting does not improve the outage rate region significantly. Hence, we do not consider that strategy. As it is not appropriate to mention about the asymptotic performance in the quasi-static fading case, we validate our code designs using only finite block length code simulations.

First we consider a Rayleigh fading IC with parameters $S N R_{1}=8 \mathrm{~dB}, S N R_{2}=S N R_{1}-1 \mathrm{~dB}, I N R_{1}=S N R_{1}+$ $3 \mathrm{~dB}$ and $I N R_{2}=S N R_{1}+2 \mathrm{~dB}$. We consider an outage probability of $P_{o}=0.1$. Optimized degree distributions with SUD are given in Table V. We also perform code design for the case of two transmit antennas considering an IC with the same channel parameters. The resulting designed degree distributions are provided in Table VI.

In the second example, we consider another Rayleigh fading IC with parameters $S N R_{1}=5 \mathrm{~dB}, S N R_{2}=S N R_{1}, I N R_{1}=$ $I N R_{2}=S N R_{1}+3 \mathrm{~dB}$ and and an outage probability of $P_{o}=$ 0.1 . Optimized degree distributions for flexible decoding are given in Table VII.

\section{Finite Block Length Results}

We now provide finite block length performance of our designed codes. We set the maximum number of iterations to 250 and 50 for block lengths greater than $2.5 \mathrm{k}$ and less than $2.5 \mathrm{k}$, respectively. We use the random construction method to build the parity check matrices [18].

In Fig. 8 the performance of the designed codes over fast Rayleigh fading IC with $10 \mathrm{k}$ block length is given. We note that the BER results are for the messages with the worst error rate (bottleneck). We observe that the optimized codes provide significant performance improvements over the P2P ones. We have also experimented with different iteration numbers and observed that, compared with the P2P codes, the designed codes reduce the required maximum iteration numbers, specifically, for the above examples from 250 to 40 for SUD and 120 for HK at a BER of $10^{-4}$.

Finite block length performance of the designed codes over quasi-static Rayleigh fading IC with SUD is depicted in Fig. 9 and Table VIII. For comparison, we also show the performance of P2P codes. The results suggest that, at an FER of 0.1 , our codes with block lengths $10 \mathrm{k}$ operate about $0.55 \mathrm{~dB}$ away from the outage limit. We also observe that the designed codes outperform the P2P codes by about $0.35 \mathrm{~dB}$. The FER performance of designed codes for the Alamouti transmission scheme is depicted in Fig. 10, indicating that, at an FER of $10^{-2}$, the designed codes with block lengths $10 \mathrm{k}$ and $2 \mathrm{k}$ operate about 0.5 and $0.8 \mathrm{~dB}$ away from the outage limit, respectively. We also deduce that the designed codes offer a performance enhancement up to $0.7 \mathrm{~dB}$ when compared to the P2P codes with the same block length. However, we also notice that the designed codes for the two antenna case have almost the same performance with the ones designed for single antenna case.

Finally, the performance of the designed codes for quasi-static Rayleigh fading IC with flexible decoding is depicted in Fig. 11 and Table IX. It is observed that the outage probability for flexible decoding is smaller than those for the TIN and SUD. In addition, the desired outage probability is achieved about $0.65 \mathrm{~dB}$ away from the target $S N R$ indicating the efficacy of our designs. The superiority of the optimized codes are observed in this example, as well. We also report that all of our designed codes provide better performance than the naive time-sharing between the users.

We further note that, while SUD scheme requires two component LDPC decoders and a state node, TIN scheme requires only a single component LDPC decoder. Hence the decoding complexity for the SUD scheme is more than twice of the decoding complexity for TIN. On the other hand, complexity of the flexible decoding scheme is between those for SUD and TIN. For the specific example we consider, at an $S N R_{1}$ of $5 \mathrm{~dB}$, only in $4.9 \%$ of the channel realizations the flexible receiver performs joint decoding, making the complexity very close to that of TIN scheme. 
TABLE III

OPTIMIZED DEgREe DistribUtions FOR HK CODING UNDER FAST FADING

\begin{tabular}{|c|c|c|c|c|c|c|c|c|c|c|c|}
\hline Msg. & $\mathrm{R}$ & $d_{c}$ & $\lambda_{2}$ & $\lambda_{3}$ & $\lambda_{4}$ & $\lambda_{9}$ & $\lambda_{10}$ & $\lambda_{19}$ & $\lambda_{20}$ & $\lambda_{49}$ & $\lambda_{50}$ \\
\hline \hline$U_{1}$ & 0.3085 & 6 & 0.247233 & 0.160895 & 0.136835 & 0.073672 & 0.142451 & 0.047803 & 0.025683 & 0.094171 & 0.071258 \\
\hline$W_{1}$ & 0.0915 & 3 & 0.51823 & 0.195265 & 0.111174 & 0.08633 & 0.030592 & 0.009713 & 0.024965 & 0.011482 & 0.012248 \\
\hline$U_{2}$ & 0.1487 & 4 & 0.368714 & 0.128519 & 0.172771 & 0.098244 & 0.079759 & 0.025089 & 0.016697 & 0.102447 & 0.007761 \\
\hline$W_{2}$ & 0.206 & 5 & 0.28792 & 0.132107 & 0.159936 & 0.085127 & 0.089978 & 0.014282 & 0.000788 & 0.179696 & 0.050166 \\
\hline \hline$U_{1}$ & 0.2045 & 5 & 0.280208 & 0.132278 & 0.179354 & 0.103725 & 0.041653 & 0.00182 & 0.045874 & 0.014092 & 0.200995 \\
\hline$W_{1}$ & 0.1713 & 4 & 0.376407 & 0.177955 & 0.11046 & 0.158397 & 0.055449 & 0.004026 & 0.027599 & 0.004899 & 0.084808 \\
\hline$U_{2}$ & 0.1713 & 4 & 0.365051 & 0.187842 & 0.12563 & 0.140829 & 0.061966 & 0.002475 & 0.026849 & 0.044926 & 0.044431 \\
\hline$W_{2}$ & 0.2045 & 5 & 0.285428 & 0.131186 & 0.167797 & 0.114655 & 0.034684 & 0.003364 & 0.043889 & 0.174081 & 0.044915 \\
\hline$U_{1}$ & 0.3728 & 7 & 0.231534 & 0.147394 & 0.113944 & 0.179444 & 0.072756 & 0.00268 & 0.064363 & 0.117768 & 0.070117 \\
\hline$W_{1}$ & 0.057 & 3 & 0.507098 & 0.179974 & 0.090527 & 0.088258 & 0.031726 & 0.033972 & 0.044318 & 0.011838 & 0.012288 \\
\hline$W_{2}$ & 0.3224 & 6 & 0.268153 & 0.097446 & 0.205879 & 0.071591 & 0.142721 & 0.034838 & 0.00844 & 0.095648 & 0.075284 \\
\hline
\end{tabular}

TABLE IV

DeCoding Thresholds In Terms OF $S N R_{1}$ FOR HK CODING Under FAST FADING

\begin{tabular}{|c|c|c|c|c|c|}
\hline $\begin{array}{c}\text { Rate pair } \\
\left(R_{1}, R_{2}\right)\end{array}$ & $\begin{array}{c}\text { P2P } \\
\text { code ensemble }\end{array}$ & $\begin{array}{c}\text { Optimized } \\
\text { code ensemble }\end{array}$ & $\begin{array}{c}\text { Achievable } \\
\text { limit (SUD) }\end{array}$ & $\begin{array}{c}\text { Achievable } \\
\text { limit (TIN) }\end{array}$ & $\begin{array}{c}\text { Achievable } \\
\text { limit }(\mathrm{HK})\end{array}$ \\
\hline$(0.4,0.3547)$ & $-1.84 \mathrm{~dB}$ & $-2.18 \mathrm{~dB}$ & $-1.55 \mathrm{~dB}$ & $-2.05 \mathrm{~dB}$ & $-2.63 \mathrm{~dB}$ \\
\hline$(0.3758,0.3758)$ & $-1.91 \mathrm{~dB}$ & $-2.15 \mathrm{~dB}$ & $-1.97 \mathrm{~dB}$ & $-2.12 \mathrm{~dB}$ & $-2.60 \mathrm{~dB}$ \\
\hline$(0.4298,0.3224)$ & $-2.05 \mathrm{~dB}$ & $-2.22 \mathrm{~dB}$ & $-0.98 \mathrm{~dB}$ & $-1.45 \mathrm{~dB}$ & $-2.54 \mathrm{~dB}$ \\
\hline
\end{tabular}

TABLE V

OPTIMIZED DEGREe Distributions For SUD AND QUASI-STATIC FADING SCENARIO

\begin{tabular}{|c|c|c|c|c|c|c|c|c|c|c|c|}
\hline Msg. & $\mathrm{R}$ & $d_{c}$ & $\lambda_{2}$ & $\lambda_{3}$ & $\lambda_{4}$ & $\lambda_{9}$ & $\lambda_{10}$ & $\lambda_{19}$ & $\lambda_{20}$ & $\lambda_{49}$ & $\lambda_{50}$ \\
\hline \hline$W_{1}$ & 0,2628 & 5 & 0,298697 & 0,144636 & 0,185787 & 0,022514 & 0,191288 & 0,058976 & 0,019543 & 0,003286 & 0,075274 \\
\hline$W_{2}$ & 0,2155 & 5 & 0,28608 & 0,12891 & 0,176329 & 0,07531 & 0,112404 & 0,021402 & 0,001853 & 0,148091 & 0,049622 \\
\hline \hline$W_{1}$ & 0,3642 & 7 & 0,217104 & 0,166379 & 0,11869 & 0,162814 & 0,05443 & 0,001543 & 0,058521 & 0,17184 & 0,048678 \\
\hline$W_{2}$ & 0,102 & 4 & 0,324211 & 0,195939 & 0,128127 & 0,099471 & 0,029218 & 0,001508 & 0,013505 & 0,130795 & 0,077227 \\
\hline \hline$W_{1}$ & 0.1314 & 4 & 0.357801 & 0.119989 & 0.19361 & 0.093057 & 0.04632 & 0.003388 & 0.053987 & 0.076639 & 0.055209 \\
\hline$W_{2}$ & 0.3153 & 6 & 0.260758 & 0.121757 & 0.18376 & 0.102342 & 0.084807 & 0.024197 & 0.031441 & 0.022821 & 0.168117 \\
\hline
\end{tabular}

TABLE VI

Optimized Degree Distributions For Alamouti TRANSMISSION AND QUASI-STATIC FADING SCENARIO

\begin{tabular}{|c|c|c|c|c|c|c|c|c|c|c|c|}
\hline Msg. & $\mathrm{R}$ & $d_{c}$ & $\lambda_{2}$ & $\lambda_{3}$ & $\lambda_{4}$ & $\lambda_{9}$ & $\lambda_{10}$ & $\lambda_{19}$ & $\lambda_{20}$ & $\lambda_{49}$ & $\lambda_{50}$ \\
\hline \hline$W_{1}$ & 0.2654 & 5 & 0.307358 & 0.131746 & 0.190356 & 0.077811 & 0.119491 & 0.030343 & 0.067058 & 0.047555 & 0.028282 \\
\hline$W_{2}$ & 0.2176 & 5 & 0.293478 & 0.115053 & 0.181472 & 0.131295 & 0.040833 & 0.050643 & 0.003052 & 0.016766 & 0.167408 \\
\hline
\end{tabular}

TABLE VII

Optimized Degree Distributions for FleXible Decoding AND Quasi-STATIC Fading Scenario

\begin{tabular}{|c|c|c|c|c|c|c|c|c|c|c|c|}
\hline Msg. & $\mathrm{R}$ & $d_{c}$ & $\lambda_{2}$ & $\lambda_{3}$ & $\lambda_{4}$ & $\lambda_{9}$ & $\lambda_{10}$ & $\lambda_{19}$ & $\lambda_{20}$ & $\lambda_{49}$ & $\lambda_{50}$ \\
\hline \hline$W_{1}$ & 0.204 & 5 & 0.275952 & 0.18641 & 0.093157 & 0.164057 & 0.040362 & 0.007804 & 0.017464 & 0.034803 & 0.17999 \\
\hline$W_{2}$ & 0.204 & 5 & 0.275952 & 0.18641 & 0.093157 & 0.164057 & 0.040362 & 0.007804 & 0.017464 & 0.034803 & 0.17999 \\
\hline \hline$W_{1}$ & 0.255 & 5 & 0.30101 & 0.137732 & 0.182577 & 0.063301 & 0.136805 & 0.046525 & 0.019292 & 0.068336 & 0.044422 \\
\hline$W_{2}$ & 0.15 & 4 & 0.354227 & 0.159814 & 0.16456 & 0.087873 & 0.086675 & 0.021908 & 0.016184 & 0.028572 & 0.080189 \\
\hline \hline$W_{1}$ & 0.15 & 4 & 0.354227 & 0.159814 & 0.16456 & 0.087873 & 0.086675 & 0.021908 & 0.016184 & 0.028572 & 0.080189 \\
\hline$W_{2}$ & 0.255 & 5 & 0.30101 & 0.137732 & 0.182577 & 0.063301 & 0.136805 & 0.046525 & 0.019292 & 0.068336 & 0.044422 \\
\hline
\end{tabular}

\section{Structured Code Constructions}

For moderate block lengths $(\approx 2 \mathrm{k})$, randomly constructed codes can suffer from presence of short cycles in their Tanner graphs resulting in high error floors [14], [19]. Protograph based codes are structured codes enjoying a lifted graph from a so called base graph or protograph [9], [20]. In [21], a construction method was proposed utilizing non-commuting permuta- tion matrices and non-abelian groups. It was shown that the resulting code has no short cycles. A non-abelian group of order $m=p q$ is characterized by $(p, q, s)$ where $p$ and $q$ are prime numbers, $\bmod (q, p-1)=0$, and $\bmod \left(s^{q}, p\right)=1$. Here, $\bmod$ is the modulo operator. In the following, we construct parity check matrices utilizing the method given in [21], and evaluate the performance of the optimized LDPC codes with moderate block lengths over fast fading ICs. 


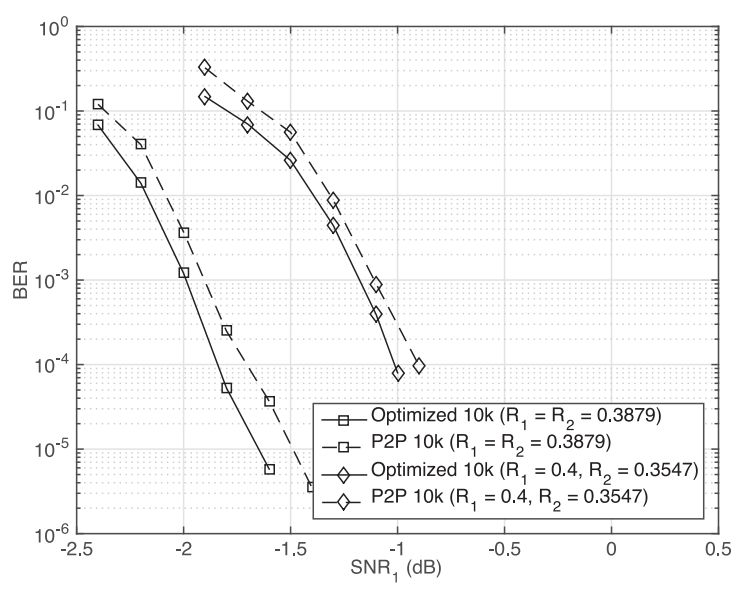

Fig. 8. Performance results with SUD and HK coding.

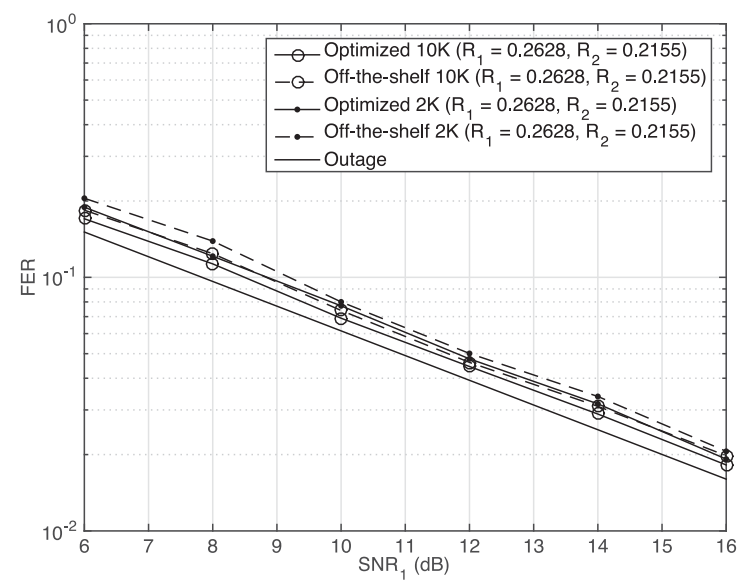

Fig. 9. Performance results with SUD.

TABLE VIII

$S N R_{1}$ FOR ACHIEVING 0.1 FER WITH SUD UNDER QUASI-STATIC FADING

\begin{tabular}{|c|c|c|c|}
\hline $\begin{array}{c}\text { Rate pair } \\
\left(R_{1}, R_{2}\right)\end{array}$ & $\begin{array}{c}\text { P2P } \\
10 \mathrm{k} \text { code }\end{array}$ & $\begin{array}{c}\text { Optimized } \\
10 \mathrm{k} \text { code }\end{array}$ & $\begin{array}{c}\text { Outage } \\
\text { (SUD) }\end{array}$ \\
\hline$(0.2628,0.2155)$ & $8.85 \mathrm{~dB}$ & $8.5 \mathrm{~dB}$ & $7.8 \mathrm{~dB}$ \\
\hline$(0.3642,0.102)$ & $8.75 \mathrm{~dB}$ & $8.4 \mathrm{~dB}$ & $7.95 \mathrm{~dB}$ \\
\hline$(0.1314,0.3153)$ & $8.7 \mathrm{~dB}$ & $8.35 \mathrm{~dB}$ & $7.85 \mathrm{~dB}$ \\
\hline
\end{tabular}

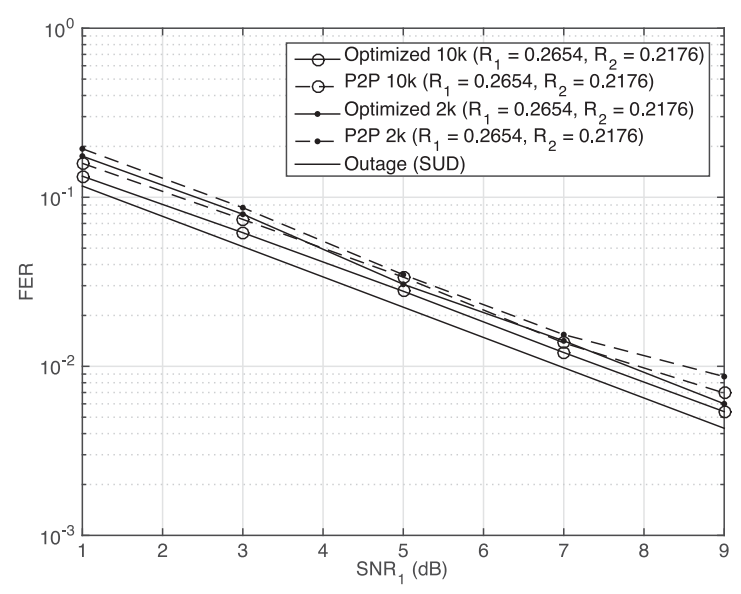

Fig. 10. Performance results with SUD and two transmit antennas.

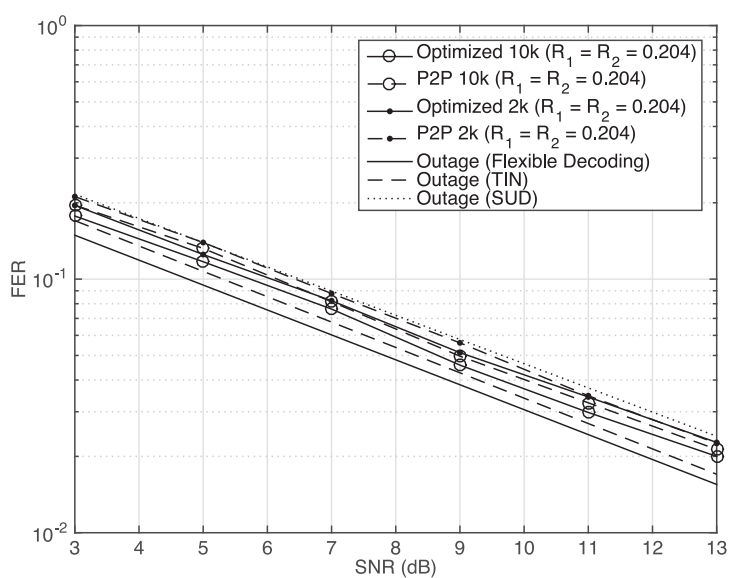

Fig. 11. Performance results with flexible decoding.

TABLE IX

$S N R$ FOR ACHIEVING 0.1 FER WITH FLEXABLE DECODING UNDER QUASI-STATIC FADING

\begin{tabular}{|c|c|c|c|c|c|}
\hline $\begin{array}{c}\text { Rate pair } \\
\left(R_{1}, R_{2}\right)\end{array}$ & $\begin{array}{c}\text { P2P } \\
10 \mathrm{k} \text { code }\end{array}$ & $\begin{array}{c}\text { Optimized } \\
10 \mathrm{k} \text { code }\end{array}$ & $\begin{array}{c}\text { Outage } \\
(\mathrm{SUD})\end{array}$ & $\begin{array}{c}\text { Outage } \\
(\text { TIN) }\end{array}$ & $\begin{array}{c}\text { Outage } \\
\text { (Flexable) }\end{array}$ \\
\hline$(0.204,0.204)$ & $6.15 \mathrm{~dB}$ & $5.75 \mathrm{~dB}$ & $6.5 \mathrm{~dB}$ & $5.3 \mathrm{~dB}$ & $4.75 \mathrm{~dB}$ \\
\hline$(0.255,0.15)$ & $5.9 \mathrm{~dB}$ & $5.6 \mathrm{~dB}$ & $6.6 \mathrm{~dB}$ & $5.35 \mathrm{~dB}$ & $4.85 \mathrm{~dB}$ \\
\hline$(0.15,0.255)$ & $5.9 \mathrm{~dB}$ & $5.6 \mathrm{~dB}$ & $6.6 \mathrm{~dB}$ & $5.35 \mathrm{~dB}$ & $4.85 \mathrm{~dB}$ \\
\hline
\end{tabular}

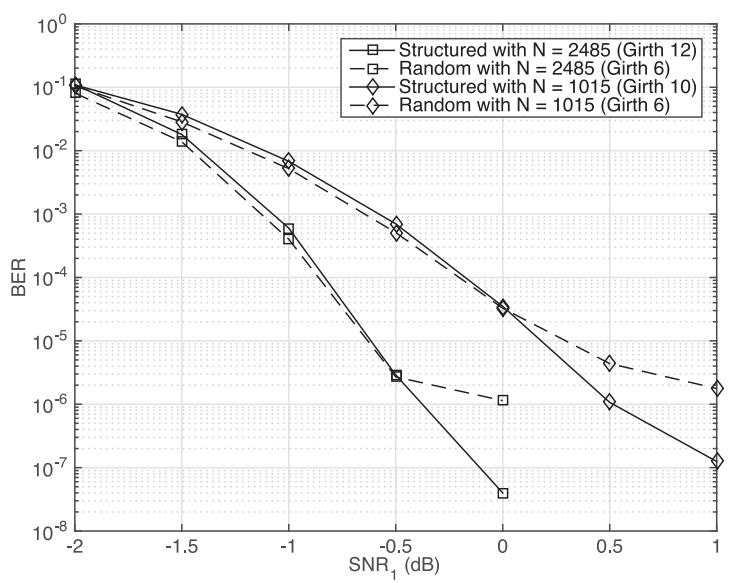

Fig. 12. BER results for the structured and random codes.

We consider $S N R_{2}=S N R_{1}+0.5 \mathrm{~dB}$ and $I N R_{i}=$ $S N R_{i}+0.75 \mathrm{~dB}$ for $i=1,2$. We fix the size of the base matrix as $3 \times 5$. In order to optimize the base matrix, we consider all possible base matrices with rate 0.4 and with non-zero rows and columns. We note that for each base matrix, there possibly exists a different degree distribution. Among these base matrices, the one with the lowest decoding threshold level is picked as the protograph. Using the design procedure introduced in the previous section, we obtain optimized degree distributions for both users as $\lambda(x)=0.3077 x+0.6923 x^{2}$ and $\rho(x)=0.6154 x^{3}+0.3846 x^{4}$. Finally, we construct codes with the optimized degree distributions for block lengths $N=1015$ and $N=2485$ with non-abelian groups $(29,7,7)$ and $(71,7,20)$, respectively. Fig. 12 shows the BER performance of the random and structured codes. For the randomly constructed codes with girth 6, we observe an error floor around $10^{-6}$. However, by 
TABLE X

OPTIMIZED DEGREE DISTRIBUTIONS UTILIZED FOR COMPARISONS WITH AR4JA CODES

\begin{tabular}{|c|c|c|c|c|c|c|c|c|c|c|c|}
\hline Msg. & $\mathrm{R}$ & $d_{c}$ & $\lambda_{2}$ & $\lambda_{3}$ & $\lambda_{4}$ & $\lambda_{9}$ & $\lambda_{10}$ & $\lambda_{19}$ & $\lambda_{20}$ & $\lambda_{49}$ & $\lambda_{50}$ \\
\hline \hline$W_{1}$ & 0.5 & 9 & 0.20044 & 0.213231 & 0.093849 & 0.116405 & 0.018885 & 0.133335 & 0.037869 & 0.023627 & 0.162357 \\
\hline$W_{2}$ & 0.5 & 9 & 0.20044 & 0.213231 & 0.093849 & 0.116405 & 0.018885 & 0.133335 & 0.037869 & 0.023627 & 0.162357 \\
\hline
\end{tabular}

using the protograph based construction method, we generate codes with girth equal to or larger than 10. For the structured code with girth 10 , an error floor occurs around $10^{-7}$, and no error floor is observed for girth 12 all the way down to $5 \times 10^{-8}$.

Finally, we consider a fast Rayleigh fading IC with $S N R_{i}=$ $I N R_{i}$ for $i=1,2$. We utilize the SUD scheme and a code rate of $1 / 2$ for both users. In order to show the performance of our designed codes in comparison with the state-of-the-art, we consider powerful AR4JA codes taken from [22]. The designed degree distributions are provided in Table X. Our simulations show that, for block sizes of $N=2048$ and $N=8192$, AR4JA codes achieve a BER of $10^{-4}$ at $S N R_{1}$ of $1.75 \mathrm{~dB}$ and $1.3 \mathrm{~dB}$, respectively. On the other hand, our designed codes achieve the same error rate at an $S N R_{1}$ of $1.2 \mathrm{~dB}$ and $0.6 \mathrm{~dB}$, respectively.

\section{CONCLUSION}

In this paper, we study the problem of code design for two user (fast and quasi-static) fading ICs. We implement two coding schemes, namely, SUD and HK. We derive the stability conditions for the degree distributions of LDPC code ensembles for both schemes under both fading scenarios. We also propose a modified EXIT chart analysis to estimate the decoding threshold of the LDPC code ensembles when multiple messages are jointly decoded. Furthermore, we investigate the receiver and code design for the case of two transmit antennas for each user. We evaluate the performance of the proposed code design method via examples and compare it with the information theoretical achievable limits. We observe that, a performance close to the achievable SNR limits can be attained for the fast fading scenario with both SUD and HK coding. For quasi-static fading, we perform code design for SUD and flexible decoding schemes. For both decoding approaches, a performance close to the outage limit is attained. We also observe that by performing flexible decoding, we can obtain an improvement in the outage. For all the instances, we notice that improvements over the P2P optimal codes can be attained. Finally, we consider structured code construction via protographs and obtain results with lower error floors.

\section{APPENDIX}

\section{A DERIVATION OF THE BHATTACHARYYA CONSTANT FOR TWO-USER CHANNEL}

In this appendix we provide a derivation of the Bhattacharyya constant of channel with $f_{Y \mid x_{1}}(y) \sim h_{1} x_{1}+h_{2} x_{2}+N$, where $x_{2} \in\{ \pm 1\}$ and $N \sim \mathcal{C N}\left(0, \sigma^{2}\right)$. The LLR of the observed signal can be written as in (31), shown at the bottom of this page. As there is no information of the signal $x_{2}$ at the receiver,

$$
\begin{aligned}
& P\left(x_{2}=1\right)=\frac{1}{2} \text {. Hence we have } \\
& \operatorname{LLR}(y)=\log \left(\frac{\exp \left(-\frac{\left\|y-h_{1}-h_{2}\right\|^{2}}{\sigma^{2}}\right)+\exp \left(-\frac{\left\|y-h_{1}+h_{2}\right\|^{2}}{\sigma^{2}}\right)}{\exp \left(-\frac{\left\|y+h_{1}+h_{2}\right\|^{2}}{\sigma^{2}}\right)+\exp \left(-\frac{\left\|y+h_{1}+h_{2}\right\|^{2}}{\sigma^{2}}\right)}\right) .
\end{aligned}
$$

After some manipulations, the $L L R(y)$ can be written as

$$
\operatorname{LLR}(y)=\frac{4 y h_{1}}{\sigma^{2}}+\log \left(\frac{\cosh \left(\left(y-h_{1}\right) \star \frac{2 h_{2}}{\sigma^{2}}\right)}{\cosh \left(\left(y+h_{1}\right) \star \frac{2 h_{2}}{\sigma^{2}}\right)}\right)
$$

where the operator $\star$ is defined as $a \star b=\operatorname{Re}(a) \operatorname{Re}(b)+$ $\operatorname{Im}(a) \operatorname{Im}(b)$. In order to derive the Bhattacharyya constant of the channel $\mathcal{B}\left(P_{L}\right)$, we follow the approach developed in [8]. Defining $P_{L}$ as the $L L R$-density of the channel output, we calculate

$$
\begin{aligned}
\mathcal{B}\left(P_{L}\right) & =\int_{\mathbb{R}} P_{L}(l) \exp \left(-\frac{l}{2}\right) d l \\
& =\int_{\mathbb{C}} P_{Y}(y) \exp \left(-\frac{L(y)}{2}\right) d y
\end{aligned}
$$

where $P_{Y}(y)$ is the PDF of the channel output. Since

$$
\begin{aligned}
P_{Y}\left(y \mid x_{1}\right)= & \frac{1}{2 \pi \sigma^{2}}\left(\exp \left(-\frac{\left\|y-h_{1}-h_{2}\right\|^{2}}{\sigma^{2}}\right)\right. \\
& \left.+\exp \left(-\frac{\left\|y-h_{1}+h_{2}\right\|^{2}}{\sigma^{2}}\right)\right),
\end{aligned}
$$

we obtain the following expression after simplifications

$$
\begin{aligned}
\mathcal{B}\left(P_{L}\right)= & \int_{\mathbb{C}} \frac{1}{\pi \sigma^{2}} \exp \left(-\frac{\|y\|^{2}}{\sigma^{2}}\right) \exp \left(-\frac{\left\|h_{1}\right\|^{2}}{\sigma^{2}}-\frac{\left\|h_{2}\right\|^{2}}{\sigma^{2}}\right) \\
& \times \sqrt{\cosh \left(\left(y+h_{1}\right) \frac{2 h_{2}}{\sigma^{2}}\right) \cosh \left(\left(y-h_{1}\right) \frac{2 h_{2}}{\sigma^{2}}\right)} d y .
\end{aligned}
$$

In a more convenient form, the Bhattacharyya constant of the channel can be expressed as in (37), shown at the top of the next page, where $N_{1}, N_{2} \sim \mathcal{N}\left(0, \frac{\sigma^{2}}{2}\right)$.

$$
\operatorname{LLR}(y)=\frac{P\left(y \mid x_{1}=1\right)}{P\left(y \mid x_{1}=-1\right)}=\log \left(\frac{\frac{1}{\pi \sigma^{2}} \exp \left(-\frac{\left\|y-h_{1}-h_{2}\right\|^{2}}{\sigma^{2}}\right) P\left(x_{2}=1\right)+\frac{1}{\pi \sigma^{2}} \exp \left(-\frac{\left\|y-h_{1}+h_{2}\right\|^{2}}{\sigma^{2}}\right) P\left(x_{2}=-1\right)}{\frac{1}{\pi \sigma^{2}} \exp \left(-\frac{\left\|y+h_{1}-h_{2}\right\|^{2}}{\sigma^{2}}\right) P\left(x_{2}=1\right)+\frac{1}{\pi \sigma^{2}} \exp \left(-\frac{\left\|y+h_{1}+h_{2}\right\|^{2}}{\sigma^{2}}\right) P\left(x_{2}=-1\right)}\right)
$$




$$
\mathcal{B}\left(P_{L}\right)=E_{N_{1}, N_{2}}\left[\exp \left(-\frac{\left\|h_{1}\right\|^{2}}{\sigma^{2}}-\frac{\left\|h_{2}\right\|^{2}}{\sigma^{2}}\right) \sqrt{\frac{\cosh \left(\frac{4 N_{1} \operatorname{Re}\left(h_{2}\right)+4 N_{2} \operatorname{Im}\left(h_{2}\right)}{\sigma^{2}}\right)+\cosh \left(\frac{4 R e\left(h_{1}\right) \operatorname{Re}\left(h_{2}\right)+4 \operatorname{Im}\left(h_{1}\right) \operatorname{Im}\left(h_{2}\right)}{\sigma^{2}}\right)}{2}}\right] .
$$

\section{REFERENCES}

[1] T. Han and K. Kobayashi, "A new achievable rate region for the interference channel," IEEE Trans. Inf. Theory, vol. IT-27, no. 1, pp. 49-60, Jan. 1981.

[2] A. El Gamal and Y. H. Kim, Network Information Theory. Cambridge, U.K: Cambridge Univ. Press, 2011.

[3] A. Bennatan, S. Shamai, and A. R. Calderbank, "Soft-decoding-based strategies for relay and interference channels: Analysis and achievable rates using LDPC codes," IEEE Trans. Inf. Theory, vol. 60, no. 4, pp. 19772009, Apr. 2014.

[4] L. Lin et al., "An information-spectrum approach to the capacity region of the interference channel," Entropy, vol. 19, no. 6, Jun. 2017, Art. no. 270.

[5] S. Sharifi, A. K. Tanc, and T. M. Duman, "Implementing the HanKobayashi scheme using low density parity check codes over Gaussian interference channels," IEEE Trans. Commun., vol. 63, no. 2, pp. 337-350, Feb. 2015.

[6] M. Zheng et al., "Polar coding strategies for the interference channel with partial-joint decoding," IEEE Trans. Inf. Theory, to be published. doi: 10.1109/TIT.2018.2878453.

[7] T. Richardson and S. Kudekar, "Design of low-density parity check codes for 5G new radio," IEEE Commun. Mag., vol. 56, no. 3, pp. 28-34, Mar. 2018.

[8] T. J. Richardson, M. A. Shokrollahi, and R. L. Urbanke, "Design of capacity-approaching irregular low-density parity-check codes," IEEE Trans. Inf. Theory, vol. 47, no. 2, pp. 619-637, Feb. 2001.

[9] Y. Fang, G. Bi, Y. L. Guan, and F. C. M. Lau, "A survey on protograph LDPC codes and their applications," IEEE Commun. Surveys Tut., vol. 17, no. 4, pp. 1989-2016, Fourth Quarter 2015.

[10] P. Berlin and D. Tuninetti, "LDPC codes for fading Gaussian broadcast channels," IEEE Trans. Inf. Theory, vol. 51, no. 6, pp. 2173-2182, Jun. 2005.

[11] S. Sharifi, A. K. Tanc, and T. M. Duman, "LDPC code design for the twouser Gaussian multiple access channel," IEEE Trans. Wireless Commun., vol. 15, no. 4, pp. 2833-2844, Apr. 2016.

[12] S. M. Alamouti, "A simple transmit diversity technique for wireless communications," IEEE J. Sel. Areas Commun., vol. 16, no. 8, pp. 1451-1458, Oct. 1998.

[13] E. Biglieri, J. Proakis, and S. Shamai, "Fading channels: Informationtheoretic and communications aspects," IEEE Trans. Inf. Theory, vol. 44, no. 6, pp. 2619-2692, Oct. 1998.

[14] T. Richardson, and R. Urbanke, Modern Coding Theory. Cambridge, U.K.: Cambridge Univ. Press, 2008.

[15] S. Ten Brink, "Designing iterative decoding schemes with the extrinsic information transfer chart," AEU-Int. J. Electron. Commun., vol. 54, no. 6, pp. 389-398, 2000.

[16] S. J. Johnson, Iterative Error Correction: Turbo, Low-Density ParityCheck and Repeat-Accumulate Codes. Cambridge, U.K.: Cambridge Univ. Press, 2010.

[17] H. Chong et al., "On the Han-Kobayashi region for the interference channel," IEEE Trans. Inf. Theory, vol. 54, no. 7, pp. 3188-3195, Jul. 2008 .

[18] IT++ Library. [Online]. Available: http://itpp.sourceforge.net/4.3.1/. Accessed on: Jul. 21, 2018.

[19] D. V. Nguyen, S. K. Chilappagari, M. W. Marcellin, and B. Vasic, "On the construction of structured LDPC codes free of small trapping sets," IEEE Trans. Inf. Theory, vol. 58, no. 4, pp. 2280-2302, Apr. 2012.

[20] D. Divsalar, S. Dolinar, C. R. Jones, and K. Andrews, "Capacityapproaching protograph codes," IEEE J. Sel. Areas Commun., vol. 27, no. 6 , pp. 876-888, Aug. 2009.
[21] C. A. Kelley, "Algebraic design and implementation of protograph codes using non-commuting permutation matrices," IEEE Trans. Commun., vol. 61, no. 3, pp. 910-918, Mar. 2013.

[22] Pretty Good Codes. [Online]. Available: http://pretty-good-codes.org/. Accessed on: Nov. 12, 2018.

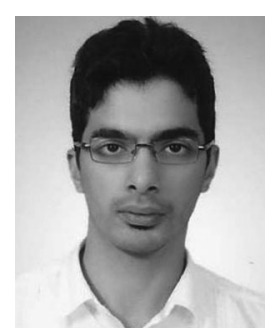

Mahdi Shakiba Herfeh (S'16) received the B.S. degree from the University of Tehran, Tehran, Iran, in 2011, and the M.S. degree from Middle East Technical University, Ankara, Turkey, in 2014. He is currently working toward the Ph.D. degree at Bilkent University, Ankara, Turkey. His research interests include various topics in information theory and wireless communications with a particular focus on coding techniques.

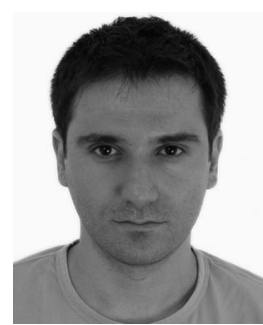

A. Korhan Tanc (S'10-M'12) received the Ph.D. degree in electronics and communications engineering from Istanbul Technical University, Istanbul, Turkey, in 2011. From 2012 to 2013, he was a Postdoctoral Researcher with the School of Electrical, Computer and Energy Engineering, Arizona State University, Tempe, AZ, USA. He is currently an Associate Professor with the Department of Biomedical Engineering, Biruni University, Istanbul, Turkey. His research interests include adaptive signal processing, communication theory, medical imaging, and multichannel processing. He is currently an Area Editor for Physical Communication (Elsevier).

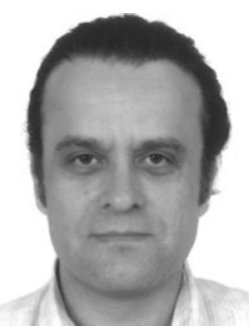

Tolga M. Duman (S'95-M'98-SM'03-F'11) received the B.S. degree from Bilkent University, Ankara, Turkey, in 1993, and the M.S. and Ph.D. degrees from Northeastern University, Boston, MA, USA, in 1995 and 1998, respectively, all in electrical engineering. He is currently a Professor with the Electrical and Electronics Engineering Department, Bilkent University. Prior to joining Bilkent University in September 2012, he was with the School of Electrical, Computer and Energy Engineering, Arizona State University, as an Assistant Professor from 1998 to 2004, an Associate Professor from 2004 to 2008, and a Professor after 2008. His current research interests are in systems, with particular focus on communication and signal processing, including wireless and mobile communications, coding/modulation, coding for wireless communications, data storage systems, and underwater acoustic communications.

Dr. Duman is a recipient of the National Science Foundation CAREER Award and the IEEE Third Millennium Medal. He was an Editor for the IEEE TRANSACTIONS ON WIRELESS COMMUNICATIONS from 2003 to 2008, the IEEE COMmUNiCATIONS SURVEYS AND TUTORIALS from 2002 to 2007, the IEEE TRANSACTIONS ON COMMUNICATIONS from 2007 to 2012, and Physical Communication (Elsevier) from 2010 to 2016. He has been the coding and information theory Area Editor for the IEEE TRANSACTIONS ON COMMUNICATIONS since 2011, an Editor for the IEEE TRANSACTIONS ON WIRELESS COMMUNICATIONS, and the Editor-in-Chief of Physical Communication (Elsevier) since 2016. 